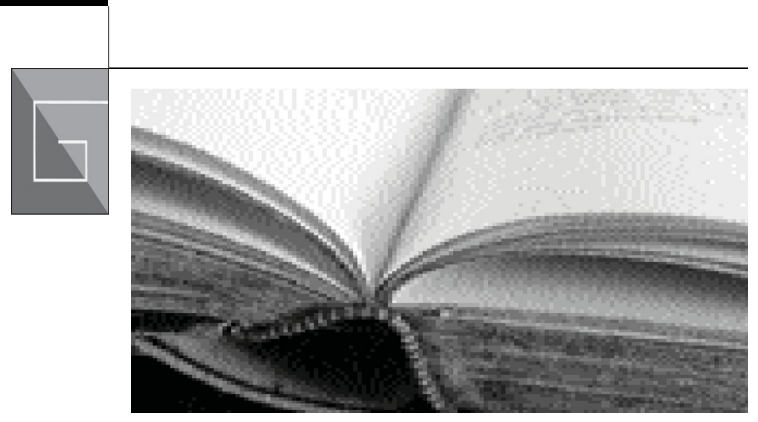

\title{
Finance immobilière $\&$ gestion de patrimoine
}

\author{
Michel Albouy \\ Éditions Economica, Nouvelle édition, 272 p., 29 euros
}

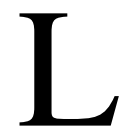

e professeur Michel Albouy est un des pionniers de la finance d'entreprise en France, tant en recherche qu'en formation. Il allie à la maîtrise de sa discipline un talent pédagogique reconnu. Parmi ses très nombreuses publications, son Décisions financières et création de valeur (Economica) figure indéniablement au sommet des ouvrages francophones du genre.

C'est encore de finance, mais de la finance appliquée à l'immobilier, dont il est question avec la nouvelle édition de Finance immobilière et gestion de patrimoine. Celle-ci actualise la version originale publiée en 2009. On y retrouve la vaste culture financière de l'auteur autant que ses qualités d'exposition et d'analyse. L'ouvrage est construit en deux parties. La première projette un regard «macro » sur le marché immobilier et les déterminants du couple rentabilité/risque de ce marché. La seconde partie zoome sur la gestion de patrimoine, les montages financiers, la fiscalité et la diversification du portefeuille.

L'entame de l'ouvrage est focalisée sur l'immobilier résidentiel : comme le titre le signifie, l'auteur traite de gestion de patrimoine et non de gestion d'actifs. L'immobilier résidentiel est au carrefour de deux logiques pour les ménages. Le logement est un bien d'équipement dont l'acquisition est conditionnée par le revenu courant du ménage, par le rapport entre ce revenu et le niveau des prix de marché, ainsi que par les conditions d'endettement (taux d'intérêt, maturité, taux d'effort acceptable). L'autre logique est celle de l'investissement d'un bien destiné non pas à l'usage du propriétaire mais à l'apport d'un revenu locatif, susceptible de couvrir le remboursement d'un emprunt le cas échéant. Dans tous les cas les questions posées à l'acquéreur sont les mêmes : Quel type d'actif immobilier? En quel lieu? Dans quelle proportion? À quel 
moment ? Avec quelle fiscalité ? À cet égard, la conclusion du livre, qui délivre huit conseils et énumère sept erreurs, ne manque ni de pertinence ni d'impertinence : elle est à encadrer par tous les conseillers en gestion de patrimoine.

L'ouvrage n'ignore pas que l'investissement immobilier concerne aussi le «tertiaire » (bureaux, commerces), la logistique, les résidences services, l'hôtellerie, etc. Un particulier peut investir dans l'immobilier non résidentiel soit directement (en acquérant les murs d'un commerce par exemple), soit en acquérant des titres : la « pierre-papier », précise l'auteur, recouvre les actions des sociétés foncières cotées, ou des parts de sociétés civiles professionnelles non cotées (SCPI). Il conviendrait d'y adjoindre les OPCI (organismes de placement collectif dans l'immobilier), proches des OPCVM dans leur fonctionnement, mais non cotés. Les fondamentaux de la finance sont largement présentés dans leur application immobilière : évaluation des actifs, mesure de rentabilité, impact du levier d'endettement, estimation du risque, gestion de portefeuille. Chaque chapitre est illustré d'exemples et propose des exercices d'application. La bibliographie de l'ouvrage est étendue. On pourrait y ajouter le Management de l'immobilier, coordonné par Denis Burckel (Vuibert), ainsi que trois sites web : celui de l'Association française des sociétés de placement immobilier (ASPIM), celui de la European Public Real Estate Association (EPRA) qui fédère les foncières cotées, et le site DVF (demandes de valeurs foncières) qui recense les prix de toutes les transactions au cours des quatre dernières années au niveau de chaque parcelle cadastrale.

L'ouvrage est bâti sur de solides fondations, il offre une architecture plaisante et invite le lecteur à cheminer sous une belle charpente : le maître d'ouvrage tient fort bien ses promesses. Amis de la finance et de l'immobilier, vous ne regretterez pas l'acquisition du « Albouy ».

Laurent BATSCH

Fondation Paris-Dauphine 